\title{
Hispanismo en Rusia y en los Países del Este: Adónde va, de dónde viene
}

\section{Vsevolod Bagno}

Arbor CLXVIII, 664 (Abril 2001), 609-621 pp.

La divulgación de los éxitos de los hispanistas de la Europa central y oriental así como sus encuentros son sumamente importantes por varias razones. En primer lugar se trata de una rama de humanidades que se desarrollaba por razones políticas en esta parte de Europa durante muchos años sin contacto autentico y profundo con el mundo hispánico y con la hispanística internacional. Al mismo tiempo se sabe muy bien que el nivel de las humanidades en los paises de la Europa del Centro y del Este ha sido en el siglo XX muy alto (se puede mencionar por ejemplo a los representantes del Circulo Lingüístico de Praga, por ejemplo a Roman Yakobson, asi como a Vladimir Propp, Mijail Bajtin, Yuri Lotman), en la mayoría de los casos a pesar de la ideología marxista-leninista. No hay que olvidar también que pensadores y cientificos occidentales de renombre como Eliade, Lukacs, Berdiaev, Todorov o Kristeva han sido de procedencia rumana, húngara, rusa o búlgara. Otro aspecto del problema es la situación actual completamente nueva con la actitud estatal respecto a la hispanística en los paises del Este en comparación con la de los años 60 o 70. No es casual que la reducción de los programas de cooperación con Cuba se reflejó en la política de los Ministerios de Educación de los paises del campo "socialista». Las dificultades que en muchas ocasiones tiene la hispanística en la parte de Europa que nos interesa son bastante lógicas en la situación, cuando se disminuye la presión ideológica y al mismo tiempo casi se anula el apoyo estatal. $\mathrm{Y}$ por último hay que mencionar el hecho de la separación de los intelectuales y por supuesto de los hispanistas de la Europa Centro-Oriental ligados de 
momento mucho mejor con sus colegas en el Occidente que unos con otros.

Por todas las razones ya mencionadas en cuanto a las perspectivas de la hispanística en los paises de la Europa central y oriental hay que subrayar la importancia del período actual, de todo punto crítico y clave. Está claro que es necesario generalizar la situación en la enseñanza y en el estudio de la lengua y la cultura hispánica, analizar los éxitos, las dificultades y las tendencias en todo este proceso.

En España se sabe poco del hispanismo en los paises del Este, aunque la hispanística no sólo en países como Polonia, Hungría o la República Checa más ligados con el Occidente, sino incluso en Rusia, comenzó a constituirse hace mas de cien años ${ }^{1}$. De Rusia se guardó la noticia de que ya en el año 1598 el zar Boris Godunov, una de las mas dramáticas figuras de la historia rusa, intentó profundizar en la enseñanza de las lenguas extranjeras para los niños y tuvo el propósito de invitar a los maestros incluso de España ${ }^{2}$. El primer filólogo,que centró su interés en la hispanística y principalmente en el estudio del teatro español del Siglo de Oro fue Dmitri Petrov, el catedrático de la Universidad Estatal de San Petersburgo, la cuna del hispanismo ruso. Sus trabajos: «Ensayos sobre el teatro de costumbres de Lope de Vega» (San Petersburgo, 1901) y "Anotaciones sobre la historia de la antigua comedia española» (San Petersburgo, 1907) en dos partes, la segunda de las cuales es la publicación del manuscrito de la comedia de Lope «Lo que pasa en una tarde») ocuparon un lugar importante en la literatura científica mundial existente sobre el teatro español. Por otro lado, hasta hoy día no han perdido su significado, y se consideran como clásicos en su ámbito los trabajos que sobre la Edad Media hiciera el conocido historiador ruso Vladimir Piskorsky. Consecuentemente se valoraban muy altamente sus investigaciones en la misma España. En el 1901 Piskorsky fue nombrado miembro correspondiente de la Real Academia de Bellas Artes de Barcelona, su trabajos («Las Cortes de Castilla en la época de transición de la Edad Media a la edad moderna (1897)» $\mathrm{y}$ «El régimen de servidumbre en Cataluña en la Edad Media» (1901)) han sido traducidos al español ${ }^{3}$.

La hispanística en Rusia con los esfuerzos de varias generaciones de estudiosos, que cada año ensanchan el diapasón de sus investigaciones, tiene en su haber éxitos de gran importancia. Sirvan como ejemplo libros tan fundamentales como el trabajo colectivo «Cultura de España» (Moscú, 1940, en ruso), «Ensayo sobre la historia de las lenguas de España» de V.Shishmarióv (Leningrado, 1941, en ruso), «Cervantes. Vida y obra» de K.Derzavin (Moscú, 1958, en ruso), «Ensayos 


\section{Hispanismo en Rusia y en los Países del Este}

sobre la historia de las relaciones literarias hispano-rusas de los siglos XVI-XIX» de M.Alekseev (Leningrado, 1964, en ruso), «Sobre el problema de la variación linguistica. El español de España y de América Latina» (Moscú, 1979, en ruso) de G.Stepanov, «Pruebas de la historia. Ensayos de la literatura española del siglo XX» de I. Terterián (Moscú, 1983, en ruso ) Por su gran aporte a la filología hispánica, M.Alekseev, G.Stepanov y I.Terterián fueron nombrados miembros extranjeros de la Real Academia Española.

Si los orígenes del interés por parte de los rusos en la cultura y la lengua españolas tienen vieja raigambre y el hispanismo ruso se enorgullece de numerosas traducciones y estupendos ensayos ${ }^{4}$, la enseñanza del español como asignatura oficial no tiene más de sesenta años. La más importante etapa en la divulgación del español en Rusia está ligada con el período de la Guerra Civil y está condicionada con las relaciones políticas entre España y la URSS. Tuvo importancia en particular la organización de los colegios para los niños españoles, niños de la guerra, que necesitaban de maestros, de manuales y de programas educativos. Es muy significativo el hecho de que hasta aquel tiempo no hubiese sido creado un centro de estudios superiores para la formación de hispanistas. En 1937 Olga Vasilieva-Shvede, entonces catedrática de la Universidad de Leningrado, escribió el primer manual soviético de lengua española ${ }^{5}$, ideado en principio para curso universitario, y convertido más tarde, debido al carácter masivo que adquirió en aquellos tiempos el interés hacia España, en curso "de iniciación». Durante muchos años precisamente la Univerisdad de Leningrado licenciaba maestros y profesores para los colegios y los centros de enseñanza superior de toda la Union Soviética.

Otra etapa muy importante, con la difusión mucho más profunda que antes de la enseñanza del español en Rusia y en general en los países del campo "socialista», también estuvo motivada por causas ideológicas. Las relaciones amistosas entre la URSS y la República de Cuba, la estrecha colaboración en el plano económico, militar y cultural necesitaron de muchos intérpretes y traductores y produjeron gran interés en el público por la lengua española.

Según el proyecto del Ministerio de Educación de la Unión Soviética en el año 1970 tendría que estudiar español un 15\% de todos los que estudiaron las lenguas extranjeras en los colegios y en los centros de enseñanza superior (igual que el alemán, en comparación con el $45 \%$ del inglés y el $25 \%$ del francés). Desgraciadamente este proyecto no se realizó, pero de todos modos el español es ahora la cuarta lengua de las lenguas extranjeras más divulgadas en Rusia. 
De gran tradición en Rusia son los estudios en la esfera del orientalismo lo que se puede aprovechar mucho más de lo que todavía se ha hecho para la investigación de las culturas hispano-árabe e hispano-hebrea. Una gran parte de los trabajos del eminente arabista ruso I.Krachkovsky estan dedicados a la cultura hispano-arabe. De los libros surgidos durante los últimos decenios sobre este tema, merece especial atención el trabajo de K.Boiko, arabista de la sección petereburguesa del Instituto del Orientalismo de la Academia de Ciencias de Rusia «Literatura histórica árabe en España (siglo VIII - primer tercio del siglo XI) (Moscú, Ed. Nauka, 1977). Hay que señalar en este plano la colaboración actual estrecha y muy fructífera de Olga Variash, historiadora moscovita, que se dedica a la España medieval y su hija Irina Variash, arabista, que investiga la cultura de Al-Andalus.

La actividad popularizadora de los hispanistas soviéticos se manifestaba no sólo en sus trabajos de investigación, o a lo largo de coloquios y congresos, sino también en la publicación de las traducciones al ruso de las obras de autores españoles o en la lengua del original. Además hay que tener en cuenta la amplitud del diapasón y de las posibilidades de las editoriales soviéticas. Todo esto respondía plenamente al profundo interés del pueblo por España, por su cultura y. su historia. En calidad de compiladores, autores de los prólogos y notas actuaban y todavía actuan tanto famosos historiadores de la literatura como maestros de la traducción literaria. Entre las numerosas traducciones de los últimos decenios se puede recordar las publicadas con una tirada de 300.000 ejemplares en la serie «Biblioteca de la Literatura Mundial», del «Poema de Mio Cid», del «Romancero», «Vida de Lazarillo de Tormes», "Historia de la vida del Buscón llamado don Pablos», de Quevedo, «El Diablo cojuelo», de Vélez de Guevara, las novelas de P.A.de Alarcón, de J.Valera, de B.Pérez Galdós, de V.Blasco Ibañez, los poemas de J.R.Jiménez, A.Machado, F.García Lorca, R.Alberti y M.Hernández.

Paradójicamente la existencia de la pared ideológica resultó ser una de las causas de la riqueza y la abundancia de libros dedicados a la enseñanza del español o la cultura española, preparados en los países del Este y publicados en sus lenguas. Es muy representativa la bibliografía de la hispanística soviética que incluye todo lo que se refiere a las monografias, artículos, material didáctico, diccionarios, tesis doctorales, y editado entre los años 1918 y 1969. La bibliografía incluye trescientos treinta y nueve trabajos ${ }^{6}$.

Por la misma causa de la existencia durante varios decenios del «telón de acero», en Rusia se editaron muchos autores españoles en 
Hispanismo en Rusia y en los Países del Este

original, necesarios para el proceso educativo, eligiendo a los que que figuraron en el catálogo de los «progresivos». Prohibiendo la edición e incluso la investigación de autores «reaccionarios», como San Juan de la Cruz, Santa Teresa, Góngora o Gracián, la censura soviética facilitaba la edición de tales obras maestras de la literatura española, como «Cancionero popular español», «Fuenteovejuna» de Lope de Vega, «Cádiz» y «Doña Perfecta» de B.Pérez Galdós, «Pepita Jimenez» de J.Valera, «Obras Escogidas» de F.García Lorca, con unas tiradas enormes según las normas occidentales. La antología bilingüe «La poesía española en las traducciones rusas 1792-1976» (Moscú, Ed. Progress, 1978) está publicada con una tirada de 38.000 ejemplares.

Dentro del panorama de la hispanística en los países de la antigua Unión Soviética, que se desarrolla actualmente por sus propios caminos, aunque continuando en muchos aspectos las tradiciones adquiridas en las aulas de las Universidades de Leningrado y de Moscú, la hispanística ucraniana, bielorusa y georgiana es de mayor alcance. Muy divulgado está el hispanismo en Ucrania, donde no sólo en Kiev, sino también en ciudades como Odesa, Lvov, Poltava, Dnepropetrovsk, Zaporozie, están trabajando en las universidades y los institutos decenas de profesores (se puede mencionar en primer lugar eminentes especialistas como P.Pomirko, Z. Getman, N.Korbozierova, M.Voronina) que dan clases de español y de literatura española, y a la vez publican manuales y sus traducciones de los autores hispánicos. En Bielorusia los estudios hispánicos se realizan desde los años 60 de nuestro siglo en la Universidad de Minsk. A propósito, durante el periodo soviético Bielorusia ha sido la más «hispanohablante» de las repúblicas de la URSS: un $4.2 \%$ de todos los que estudiaron las lenguas extranjeras estudiaban español.

El particular interés de la sociedad georgiana por España está condicionado por la teoría sobre el posible origen común de georgianos y vascos. Un gran número de trabajos de los científicos georgianos ligados con la Península Iberica está dedicado a la diversa problematica que afecta al País Vasco. Por cierto que sus autores tuvieron que exponer cuestiones tanto científicas como populares ya que, a pesar de que la literatura sobre el tema vasco que existe en idioma georgiano es bastante amplia, en ruso, durante los últimos años, se publicó solamente un contado número de trabajos. Los trabajos de los vascólogos sobre aspectos lingüísticos y etnográficos de la hipótesis vasco-caucasica se encuentran ampliamente representados en el libro «Ibérica. Cultura de los pueblos de la Península Ibérica» ${ }^{7}$.

Hay que destacar a la Fundación Duques de Soria, que realiza muy valiosas actividades y hace esfuerzos en apoyo de la hispanística 
mundial y que organizó en Salamanca, en junio de 1991, el coloquio «El español y el futuro del hispanismo ante los cambios ocurridos en los paises del Este de Europa». Participaron en él 30 representantes del hispanismo de Bulgaria, Checoslovaquia, Hungría, Polonia y Rumania y por supuesto los profesores de renombre de varias Universidades de España. De esta reunión nació una «Declaración de Salamanca», elaborada por los hispanistas allí presentes.

El Primer Simposio Internacional «Europa del Centro y del Este y el Mundo Hispánico», celebrado en la Universidad Jaguellonica de Cracovia entre el 26 y 28 de octubre de 1995 cumplía con el mismo fin. Estuvo organizado por el Instituto de Filología Románica de la antigua y famosa universidad polaca, en primer lugar por la Dra. Teresa Eminowich, junto con las Universidades de Wroclaw, de Varsovia y la Asociación de Hispanistas Polacos. No tiene nada de asombroso el que los hispanistas polacos tuvieran esta iniciativa: la hispanística en Polonia, con los esfuerzos de varias generaciones de estudiosos, que cada año ansachan el diapasón de sus investigaciones, tiene en su haber éxitos de gran importancia. Además de los hispanistas polacos en el Simposio participaron lingüístas, historiadores de la literatura, la política, la filosofía y del arte del mundo hispánico de España, Bulgaria, República Checa, Eslovaquia, Rumanía, Rusia y Ucrania.

Los materiales del Simposio se dividen en las Actas en tres grandes bloques: «Relaciones políticas, literarias y artísticas»; «Lingüística»; «Enseñanza y traducción del español». Al mismo tiempo hay que subrayar que junto con los temas tradicionales para los hispanistas del cualquier parte del mundo, donde cada uno puede traer su aportación individual, tales como la enseñanza de español como lengua extranjera, el libro tiene algunos rasgos muy peculiares. Se puede mencionar por ejemplo el tema de las culturas intermediarias (Polonia para Rusia o Francia para toda la Europa Centro-Oriental en el curso de los siglos, Rusia en algunas ocasiones en el siglo XX) que se nota en varios trabajos. Este aspecto está ligado muy estrechamente con el ambiente comparativo, desde el punto de vista muy amplio y abierto (no sólo la divulgación de la lengua y literatura española o hispanoamericana en dichos países) que ofrece buenas perspectivas. En este plano son muy representativos los artículos de Piotr Sawicki y Beata Zakes "Cartas sobre España" de Vasilii Petrovich Botkin y "El viaje a traves de España" de Jan Stella Sawicki: en la pista de un plagio literario» y de M.Pierrette Malcuzynska «Mijail Bajtin y los estudios literarios hispánicos (con una aportación bibliográfica)». En cuanto a la tesis de las coincidencias entre el pensamiento filosófico en la Europa Centro-Oriental y en Ibe- 
roamérica, propuesta y probada por Eugeniusz Gorski, me gustaría añadir mi idea de la semejanza entre otros dos modelos de las culturas fronterizas, situadas en dos zonas marginales de Europa: España y Rusia. La importancia de la colaboración muy estrecha de los colegas de nuestra zona del hispanismo europeo se prueba por la falta de las referencias rusas en otros dos trabajos del ámbito comparativo: de Beata Baczynska, «Lecturas románticas de la obra de Pedro Calderón de la Barca. Una perspectiva polaca» y de G.Rokiski Lazaro «Polonia en la poesia española del siglo XIX». El artículo de N.Balachóv y Y.Staniukovich «La unidad doble del drama "El príncipe constante" en la traducción romántica de Slovatskiy», publicado en el libro "Calderón y la cultura mundial» (Leningrado, 1986) y el libro del gran hispanista ruso de los comienzos del siglo XX D.K.Petrov «Rusia y Nikolas I en las poesias de Espronceda y Rossetti» (San Petersburgo, 1909) pudieran ser útiles para estos estudios en lo general muy interesantes e informativos.

La diversa problemática que afecta a lengua, literatura, cultura y política del mundo hispánico se reflejó en trabajos como «Confines y fronteras. El paralelo histórico a finales del siglo XX» del Embajador de Polonia en España, el profesor Jan Kieniewicz, «El personaje de la pícara española y Moll Flanders de Defoe», de la profesora Mariana Dimitrova (Sofia), «Cervantes en Rumania» de la profesora Tudora Sandru Olteanu (Bucarest), «La hispanística en Rusia» de la profesora Kseniia Lámina (San Petersburgo), «Edward Porebowicz y la lingüística española» del profesor Roman Pomirko (Lvov).

En cuanto a Rusia, en el año 1994 se celebró en Moscú en la sede de la Universidad Lingüísica el I Congreso de Hispanistas de Rusia ${ }^{8}$ patrocinado por la Embajada de España en Moscú. En el Congreso fue creada la Asociación Rusa de Hispanistas, con dos centros regionales: Moscú y San Petersburgo. Gracias a la ayuda del Ministerio de Asuntos Exteriores salieron a la luz las Actas del Congreso ${ }^{9}$. En el año 1999 también en Moscú se celebró el II Congreso de Hispanistas de Rusia.

Una de las peculiaridades de la hispanística en Rusia y en la mayoría de los países del Centro y del Este de Europa ha sido la lengua nacional como casi el único vehículo de divulgación no sólo de la cultura sino también de la lengua española. De dos peligros de aislamiento - de los colegas en otros países del mundo o del público nacional- el hispanismo ruso desde hace mucho tiempo ha elegido el primero. En general, los libros se publican en ruso, los artículos en las revistas especializadas y académicas en ruso, la enseñanza, 
por lo menos de literatura española se realiza en ruso, las tesis se defienden en ruso. El que gana es el público ruso, porque, por ejemplo, mi libro «Por los caminos del Quijote», del año 1988, salió con una tirada de 60.000 ejemplares. Por supuesto otra cara de la moneda es el hecho de que este libro sigue siendo la tierra incógnita para mis colegas hispanistas en España y en otros países. Sin duda alguna una de las tareas de nuestro hispanismo es romper esta tradición pero no por completo sino encontrando un equilibrio, huyendo de tales peligros y aprovechando ambas ventajas.

Entre las dificultades de la difusión del español en Rusia y sin duda alguna en general en los países del campo «socialista» que surgen en el periodo de tránsito hay que subrayar las que están ligadas con el proceso de la liberación del proceso educativo. Ahora no se trata de colegios y de lenguas obligatorias, y los padres tiene derecho a elegir las lenguas para la enseñanza de sus hijos, a diferencia del pasado «soviético», cuando todo funcionaba a través del orden. Por eso ahora aumenta la importancia de la propaganda. En este plano es muy representativo el ejemplo de San Petersburgo, donde en vez de disminuir la cantidad de los alumnos, aumenta, también crecen los colegios «españoles». Ahora en San Petersburgo hay ocho colegios donde el español es la única o la primera lengua extranjera. Las mismas tendencias se reflejan en la enseñanza superior, donde se notan intentos de cerrar las secciones, Departamentos o Facultades del español, o por lo menos disminuir la cantidad de los grupos. Por ejemplo en la Universidad de San Petersburgo los últimos años en vez de dos grupos hay sólo uno. Hay que añadir que este proceso no tiene nada en común con el interés por parte de los jóvenes hacia la lengua. La cantidad de los candidatos a ingresar en las secciones y los departamentos de español de las universidades y los institutos no disminuye. A propósito, la idea de enseñar la lengua y la cultura españolas por via Internet sin duda alguna abre grandes perspectivas para el futuro de la hispanística.

A la vuelta del milenio no hay que olvidar que la hispanística rusa tiene algunas misiones peculiares. Una de las mas importantes es la investigación del quijotismo ruso. La suerte que el Quijote ha corrido en Rusia no es sólo la versión nacional de un proceso cultural general, sino uno de los raros ejemplos de la historia de la cultura en que el fenomeno puramente literario de un país concreto se convierte en dominante de la vida cultural y pública de otro país, con la inevitable pérdida de muchos, por no decir la mayoría, de sus peculiaridades historico-literarias concretas. En el Quijote los rusos han visto no sólo 
una obra genial, sino toda una parábola sobre los destinos del hombre; en el protagonista de la novela, un profeta o falso profeta, el mito cuyo entorno podía servir de clave para la interpretación de los acontecimientos de la vida intelectual y pública de Rusia. Por eso deben interesarnos en igual medida traducciones, escenificaciones o ensayos, o, en otras palabras, la historia real de la recepción de la novela de Cervantes en Rusia, equiparable a la aceptación de cualquier otra obra en un idioma extranjero, por una parte, y por otra, la historia del quijotismo ruso como fenómeno cultural, las interpretaciones filosófico-psicológicas de la imagen cervantina, la interpretación creativa de los escritores rusos el mito de Don Quijote, el recurso al hombre del héroe cervantino en las polémicas de la opinión pública rusa. La distancia existente entre el protagonista y la novela, así como entre la novela y su autor, inevitable en el proceso histórico-cultural, no desmerece en lo más mínimo el valor de la novela ni del escritor, sino que amplía el espectro de interpretaciones ${ }^{10}$.

Otras de las importantes tareas para los hispanistas del Centro y del Este de Europa son la búsqueda, el estudio, la catalogación y la publicación de los manuscritos españoles que se conservan en los fondos de los archivos y bibliotecas de nuestros países. Por ejemplo, sólo en los archivos de Moscú y San Petersburgo se guardan numerosos manuscritos, los más antiguos de los cuales se remontan al siglo IX, manuscritos hispano-árabes e hispano-hebreos, cartas de Fernando el Católico y de Carlos V, la correspondencia diplomática entre las Cortes de España y Francia, que salvó un modesto funcionario de la Embajada de Rusia en París, las cartas de S.Ignacio de Loyola y de Rafael del Riego, la correspondencia de Palacio Valdés y Blasco Ibañez con sus traductoras rusas y la riquísima documentación sobre la Guerra Civil Española, innaccesible hasta el momento para los especialistas. El origen de este proyecto reside en la labor llevada a cabo por el Prof. Dr. D. Emilio Sáez Sánchez, fallecido en 1988, en San Petersburgo y Moscú, donde encontró importantes manuscritos medievales españoles. Los equipos de los historiadores madrileños y moscovitas investigaron y publicaron en el curso de los últimos años los documentos ligados con las relaciones diplomaticas entre Rusia y España ${ }^{11}$. Carlos Sáez Sánchez publicó hace poco el catálogo preparado por su padre Emilio Sáez ${ }^{12}$. Al mismo tiempo no olvidemos que, además de los incendios y las inundaciones, existen y van a existir la locura, la ignorancia y la codicia humana. Como se sabe por las famosas "Cartas desde Rusia» de Juan Valera, en la Biblioteca Imperial de San Petersburgo se guardaban los manuscritos de dramas desconocidos de Pedro Calderón de 
la Barca. El gobierno bolchevique los devolvió junto con otros manuscritos a Polonia, y, según parece, desaparecieron durante la Segunda Guerra Mundial. Hay noticias de que se puede encontrar una carta del Zar Ivan el Terrible dirigida a su contemporáneo Felipe II. Según varios testimonios, Galdós poseía cartas de Tolstoi y Turgueniev ${ }^{13}$. No se sabe dónde están ahora. Todavía no se ha encontrado la carta de Tolstoi dirigida a la redacción de la «Revista Blanca», que jugaba un papel tan importante en la novela «La voluntad» de Azorín. Todavía no se puede trabajar con el importantísimo archivo del Partido Socialista Obrero Español, traído con una valiosísima documentación española contemporánea por las tropas soviéticas desde Berlín. No falta el entusiasmo por parte de las personas dispuestas a trabajar sobre esos tesoros. Pero sin el apoyo institucional poco o nada podremos hacer.

Una de las lineas de actuación cultural y científica de los hispanistas de los países del Este debe ser el trabajo científico y de investigación realizado con la historia de la colonia española en nuestros países, en algunos casos común y que sólo se puede realizar uniendo los esfuerzos. Por ejemplo, de los hispanistas rusos y ucranianos cuando se trata de la conmemoración del Segundo Centenario de la muerte del Almirante José de Ribas (1749-1880), figura clave de las Fuerzas Armadas y destacado político de la época de Catalina II la Grande. Jose de Ribas se incorporó al servicio de Rusia en 1772. Gran estratega militar y artífice de la victoria definitiva de Rusia sobre Turquía en la costa del Mar Negro durante la guerra de 1787 y 1791, fundador en 1795 del estratégico puerto de Odesa, una de las ciudades más importantes de Ucrania y del litoral mediterráneo. José de Ribas está enterrado en San Petersburgo.

Se pueden prever tales lineas de desarrollo en el ámbito de la hispanística en los países del Centro y del Este como una colaboración con sus colegas en España mucho más estrecha que antes (desgraciadamente por causas muy conocidas los hispanistas de Bielorusia o de Rumania no pueden gozar de tales contactos con las universidades occidentales como sus colegas checos, húngaros o polacos) en la investigación de algunos proyectos concretos y peculiares, cuando el intercambio de los éxitos de distintas escuelas es sumamente importante. A mi parecer son bastente convincentes dos Coloquios Internacionales dedicados al tema «Culturas fronterizas entre Oriente y Occidente. Rusia, España» que he organizado en los años 1995 y 1999 en San Petersburgo. Han participado historiadores, antropólogos, filósofos, historiadores de la literatura, hispanistas, rusistas, arabistas, hebraistas, bizantinistas no sólo de Rusia y España, sino también de Holanda, 
Israel y Yugoslavia, lo que abre grandes perspectivas para el mejor conocimiento de la mentalidad fronteriza y de muy distintos modelos de la cultura fronteriza como España y Rusia, pero también del Oriente Próximo, los Balcanes o el Cáucaso. Por lo que se refiere a España y Rusia, la historia secular de sus respectivas culturas nos muestra el papel que ambas han desempeñado (y desempeñan) entre Oriente y Occidente. Sus respectivas posiciones geográficas las han convertido en puente, puerta, ventana o encrucijada de culturas. Pese a todas sus diferencias, estos dos casos permiten poner de manifiesto algunas regularidades características de los países fronterizos ${ }^{14}$.

Uno de los rasgos del hispanismo que viene va a ser la divulgación más amplia y profunda en España y los países hispanoamericanos de los éxitos de las humanidades de los países del Centro y del Este de Europa en el siglo XX, sin olvidar la procedencia de esta parte de Europa de pensadores y científicos de renombre como Eliade, Jakobson o Tódorov. Al parecer la única excepción es la huella muy profunda que ha dejado la obra y la teoría de Mijail Bajtin en el area del hispanismo mundial ${ }^{15}$. Por ejemplo, los mejores trabajos de los hispanistas rusos forman parte de esa crítica de inspiración bajtiniana dos monografias bastante recientes de ámbito cervantino ${ }^{16}$. Al mismo tiempo hay mucho que hacer, por ejemplo en la esfera de los estudios del folklore y las tradiciones populares del mundo hispánico apoyándose en las ideas (y no sólo en algunas citas) de Vladimir Propp.

En las nuevas condiciones, en que no funciona tal como antes el apoyo estatal al hispanismo, aparecen nuevas posibilidades inexistentes en los regímenes totalitarios. Puedo mencionar lo que conozco más: mi propia experiencia en las actividades de la Organización Social «Fundación Cervantes» de San Petersburgo que he creado en el año 1995. Sus principales programas son: El Programa «Cervantes», dedicado a la popularización en Rusia de la obra cervantina, el estudio del destino de Don Quijote y del quijotismo en Rusia; El programa «Menéndez Pidal», orientado hacia la búsqueda, el estudio y la catalogación de manuscritos españoles en Rusia; El programa «José de Ribas» - trabajo cientifico-investigador relacionado con la historia de la colonia española en San Petersburgo, la inmortalización de la memoria de los españoles que estuvieron relacionados con Rusia y Petersburgo y con su destino. Ademas, la Fundación Cervantes de San Petersburgo trabaja en las siguientes direcciones: la organización de las «Jornadas Cervantinas» anuales ${ }^{17}$; la publicación de la serie «Biblioteca de Literatura Española» ${ }^{18}$, la actividad de la «Biblioteca Hispánica» en nuestro local; la creación de la pagina WEB «El destino ruso del mito de 
Don Quijote» (www:http//quijotismo-ruso.phs.ru). La Fundacion Cervantes forma parte del «Programa de colaboración en el campo cultural y educativo entre la Federación Rusa y España para los años 1999-2001».

No podría terminar este artículo sin decir que uno de los rasgos más esenciales del hispanismo que viene va a ser la imposibilidad del tema: «Hispanismo en los países del Centro y del Este de Europa». Por lo menos por dos razones. En primer lugar, porque el hispanismo va a ser en el futuro mucho más uniforme y unido, igual que Europa en general. Y en segundo lugar porque cada uno de los países (Rumanía, Ucrania, Yugoslavia, Hungría, Armenia, etc.), a pesar de todas sus preferencias, étnicas, geográficas, culturales y ideológicas en algunos casos multiseculares, seguirá su propio camino.

\section{Notas}

1 Por ejemplo, con motivo del centenario de estudios hispánicos en Polonia, el Departamento de Estudios Ibéricos de la Universidad de Varsovia organizó los días 2-4 de diciembre de 1999 el Simposio Internacional «El hispanismo europeo a fin de siglo".

2 Véase el artículo del famoso comparatista ruso Mijail Alekseev, «Hispanística a la luz de las relaciones culturales hispano-rusas", Alekseev M. La literatura rusa $y$ el mundo romance, Leningrado, Ed. Nauka, 1985, p. 260 (en ruso).

3 Véase: L.T. Milskaya, I.S. Pychuguina, V.K. Piskorski, primer hispanista ruso, El contacto. España, vista por los historiadores soviéticos y españoles. P.I, Moscú, Editorial del Instituto de Historia Universal, 1990, p. 72-95.

4 Mijail Alekseev. Rusia y España: Una respuesta cultural, Madrid, Ed. «Hora H», 1975.

5 Uno de los primeros intentos de estudio de la lengua española en el imperio ruso fue la Gramática breve española, según las normas de la Real Academia Española, publicada por Jacob Langen (Mitava, 1811, en ruso). Más tarde apareció Material didáctico para el estudio de la lengua española, compuesto por Yakov Rut, bibliotecario de la Universidad (San Petersburgo, 1844, en ruso).

6 A.V. Suprun, N.A. Moshkovich. Hispanística en URSS. Bibliografia (1918-1969). Moscú, 1970 (en ruso).

7 Véase por ejemplo, Yu. Zitsar, «Sobre el estado actual de la lengua y la cultura vascas", Ibérica. Cultura de los pueblos de la Península Ibérica. Leningrado, Ed. Nauka, 1983, pp. 174-195 (en ruso); S. Gabunia. «Investigaciones científicas de la etnografía vasca y paralelos etnográficos vasco-caucasicos", Ibid, pp. 196-200 (en ruso); N. Sturua Problemas del vascuense en la vascología contemporánea (La revista «Fontes linguae vasconum»), Ibid, pp. 201-207 (en ruso); G. Shalamberidze "Papel de los compuestos en la estructuración de la vascológia comparada", Ibid, pp. 208-215 (en ruso).

8 En 1970, en el recinto de la Universidad de Leningrado, se celebró el I Congreso de Filología Hispánica de la Unión Soviética. Otra nueva etapa en el desarollo de la hispanística en la URSS comenzó en el año 1977 y está directamente relacionada 


\section{Hispanismo en Rusia y en los Países del Este}

con la fundación, según la decisión de la Academia de Ciencias de la URSS, de un Consejo Interdisciplinario para el Estudio de la Cultura de los Pueblos de la Península Ibérica. Para la definición de las principales directrices de investigaciones y de una planificación con vistas a futuros estudios, en 1979 se celebró en Moscú el Congreso Nacional «Problemas fundamentales del estudio de la cultura de los pueblos de la Península Ibérica».

9 Actas de la I Conferencia de Hispanistas de Rusia. Madrid, Ministerio de Asuntos Exteriores, 1995.

10 Vsevolod Bagno. El Quijote vivido por los rusos, Madrid, Ed. Diputación de Ciudad Real y Publicaciones del CSIC, 1995.

11 Véase el trabajo colectivo de los historiadores españoles y rusos basado en los materiales traídos de los archivos Rusia y España. Documentos y materiales. T.I. 1667-1799. (Moscú, 1991, en ruso); Manuel Espadas Burgos (ed.). Corpus diplomático Hispano-ruso (1667-1799), Madrid, Ministerio de Asuntos Exteriores, 1991; Rusia y España. Documentos y materiales. T.2. 1800-1917 (Moscú, 1997, en ruso).

12 Emilio Sáez, Carlos Sáez. El fondo español del archivo de la Academia de las Ciencias de San Petersburgo. Alcalá de Henares, Universidad de Alcalá de Henares, 1993.

13 H.Ch. Berkowich. «Gleanings from Galdos' correspondence», Hispania, Appleton, T.XVI, 1933, oct., p. 249.

14 Con más detalle ver: Vsevolod Bagno. «España y Rusia: dos culturas de frontera», Nueva Revista, Madrid, 1999, V.64, agosto, P.56-64; Vsevolod Bagno. Rusia y España: frontera común, Diálogo de culturas, Santiago de Compostela, Universidade de Santiago de Compostela, 1998, pp. 11-28.

15 Véase por ejemplo el artículo de M.Pierrette Malkuzynska «Mijail Bajtin y los estudios literarios hispanicos (con una aportación bibliográfica)", Ana I. Blanco / Teresa Eminowich (eds.): Europa del Centro y del Este y el Mundo Hispánico: Simposio Internacional de Hispanistas. Cracovia, 26-28 de octubre de 1995. Krakow, 1996, pp. 133-157.

16 Olga Svetlakova. "Don Quijote» de Cervantes. Problemas de la poética. San Petersburgo, Editorial de la Universidad de San Petersburgo, 1996 (en ruso); Svetlana Piskunova. "Don Quijote» y los géneros de la prosa española de los siglos XVI-XVII. Moscú, Editorial de la Universidad de Moscú, 1998 (en ruso).

17 En el abril de 1999 hemos celebrado las «XXI Jornadas Cervantinas».

18 En el año 1998 ha salido el primer volúmen - Antología de la Poesía Española Contemporánea; en al año 1999, la pieza de Lope de Vega El Gran Duque de Moscovia, por primera vez traducida al ruso; está en marcha la traducción de las poesías de Quevedo. Hemos realizado las traducciones gracias a la ayuda de la Dirección General del Libro, Archivos y Bibliotecas del Ministerio de Educación y Cultura de España. 